\title{
Über die Geschwindigkeit der Fermentreaktionen.
}

\author{
III. Mitteilung.
}

Von

R. O. Herzog.

(Aus dem chemischen Institut der Technischen Hochschule zu Karlsruhe.)

(Der Redaktion zugegangen am 23. Juni 1906.)

1. G. Bredig hat wohl zuerst mit aller Deutlichkeit ausgesprochen, daß die Katalyse durch Fermente in einem mikroheterogenen Medium ${ }^{1}$ ) stattfinde, damit war auch angedeutet, daß der Verlauf der Fermentreaktionen, welcher nicht den einfachen Formeln der chemischen Dynamik ${ }^{2}$ ) entspricht, bedingt sein könnte durch den kolloidalen Charakter der Enzyme. In der Reaktion [Wasserstoffsuperoxyd - kolloidales Platin] hatte Bredig ein geradezu ideales Modell der Fermentreaktionen gefunden. Senter, ${ }^{3}$ ) Issajew, $\left.{ }^{4}\right)$ Feitelowitz, $\left.{ }^{5}\right)$ Euler ${ }^{6}$ ) u. a. haben in den letzten Jahren die Reaktion $\mathrm{H}_{2} \mathrm{O}_{2}-\mathrm{H}_{2} \mathrm{O}_{2}$-spaltendes Ferment] ${ }^{7}$ ) untersucht, ohne (wenigstens in gewissem Konzentrationsbereich) irgend einen wesentlichen Unterschied zwischen dieser und der von Bredig untersuchten Reaktion zu finden. Es hat sich gezeigt, daß der Reaktionsverlauf mit genügender Genauigkeit durch eine Gleichung von der Form dargestellt wird, die für eine Reaktion erster Ordnung gilt.

Anders liegt der Fall aber bei allen anderen Fermentreaktionen, die bisher untersucht wurden; in allen Fällen gilt

1) Anorg. Fermente u. in den Ergebn. d. Physiologie, Bd. I, S. 134, (1902). - Die hier diskutierten Verhältnisse gelten natürlich nur für homogene Lösungen des Substrates.

2) Vgl. Tammann, Diese Zeitschrift, Bd. XVI, S. 271 (1892).

3) Zeitschrift f. physikal. Chemie, Bd. XLIV, S. 257 (1903), und Bd. LI, S. 673 (1905).

4) Diese Zeitschrift, Bd. XLII, S. 102 (1904).

5) Diss. Heidelberg 1904 .

6) Hofmeister s Beitr., Bd. VII, S. 1 (1905).

7) Vielleicht empfiehlt sich zur Abkürzung diese Schreibweise. 
die Regel, daß bei gleichbleibenden Fermentmengen der Umsatz in Prozenten der Substratkonzentration ausgedrückt mit Erhöhung der Substratmenge abnimmt. ${ }^{1}$ ) Ferner gilt in vielen Fällen für den Reaktionsverlauf nicht die einfache Formel

$$
\frac{d x}{d t}=k(a-x)
$$

sondern es bedarf gewisser Korrektionsglieder, um den Vorgang $z u$ beschreiben. Zunächst hat V. Henri ${ }^{2}$ ) gezeigt, daß der Reaktion [Rohrzucker - Invertin] die Formel

$$
\frac{d x}{d t}=\left(k_{1}+k_{2} x\right)(a-x)
$$

genügt; dann habe ich die Funktion

$$
\left.\frac{d x}{d t}=\left(k_{1}-k_{2} x\right)(a-x) \quad 3\right)
$$

für die Reaktion [Glukoside - Emulsin] gegeben. ${ }^{3}$ ) Beide Formeln sind auch für andere Reaktionen anwendbar. Wie ersichtlich, sind sie aus der Annahme abgeleitet, daß die entstehenden Reaktionsprodukte auf die Geschwindigkeit des Umsatzes Einfluß nehmen, ein Vorgang, auf den schon Tam mann ${ }^{4}$ ) hingewiesen hat.

Henri hat sehr bald seine erste Formel (2) aufgegeben, die zur Konsequenz führen mußte, eine Funktion $F(k$, a) $z u$ suchen, ${ }^{5}$ ) und nahm im Anschluß an einen Gedanken Bodensteins Bindungen zwischen Substrat sowie Spaltungsprodukten mit dem Ferment an, die durch das Massenwirkungsgesetz beherrscht sein sollten, ${ }^{6}$ ) eine Annahme, die für kolloidale Lösungen Schwierigkeiten bot. Herr Henri hat weiterhin auch diesen Weg verlassen und stellt sich jetzt vor: ${ }^{7}$ ) «Wir haben

1) Die Gültigkeit der Regel ist nachgewiesen für die Reaktionen: [Rohrzucker - Invertin], [Maltose - Maltase], [Milchzucker - Laktase], [Stärke - Diastase], [Glukoside - Emulsin], [Harnstoff-Urease], [Protein Proteolytische Enzyme], [Hexosen - Zymase], [Fette - Lipase].

2) Zeitschrift f. physik. Chemie, Bd. XXXIX, S. 194 (1902).

$\left.{ }^{8}\right)$ Konink. Akad. v. Wetensch. te Amsterdam. Sitzungsber. vom 21. Oktober 1903.

4) $1 . \mathrm{c}$.

5) Vgl. Henri, Zeitschr. f. physik. Chemie, Bd. XXXIX, S. 215 (1902).

6) Lois générales. Vgl. auch Diese Zeitschrift, Bd. XLV, S. 437 (1905).

${ }^{7)}$ Zeitschrift f. physik. Chemie, Bd. LI, S. 19 (1905). 
drei Hauptbestandteile, in die sich eine Enzymwirkung einteilen läßt: 1. das Verteilungsgesetz zwischen dem Kolloid und der Lösung; 2. die Geschwindigkeit, mit der sich diese Verteilung einstellt; 3. die Reaktionsgeschwindigkeit selbst. Dazu müssen wir noch hinzufügen den Einfluß anderer Körper (unter denen die Reaktionsprodukte stehen) auf diese drei Vorgänge.»1) Die mathematische Formulierung ${ }^{2}$ ) führt zur Gleichung:

$\frac{a-x}{a}=\frac{1}{k_{2}-k_{1}}\left[\left(k_{2}-\frac{1}{1+m a}\right) e^{-k_{1} t}-\left(k_{1}-\frac{1}{1+m a}\right) e^{-k_{2} t}\right]$

Der Verfasser bemerkt dazu aber, «daß dies nur als erste Skizze aufgefaßt werden kann, die einer näheren gründlicheren Bearbeitung bedarf ${ }^{3}$ )

Außer Henri haben in den letzten Jahren noch Barendrecht ${ }^{4}$ ) und Vis ser ${ }^{5}$ ) versucht, die Geschwindigkeit der Fermentreaktionen mathematisch $\mathrm{zu}$ beschreiben. Barendrecht entwickelt die Vorstellung, die Enzyme bewirkten durch Strahlung die Spaltung der Stoffe. Er erhält die Gleichung:

$$
\begin{aligned}
& k=\frac{a}{t}\left[(1-n) y+n \ln \frac{1}{1-y}\right] \\
& \left(k \text { und } n \text { Konstante, } y=\frac{a-x}{x}\right)
\end{aligned}
$$

Herr Visser ${ }^{6}$ ) zeigt, daß die Gleichung identisch ist mit der von Bodenstein 7 gegebenen, und von dieser hat $\mathrm{Henr}^{8}$ ) nachgewiesen, daß sie nicht ausreicht.

Visser ${ }^{6}$ ) geht davon aus, daß die Fermentreaktionen zu einem Gleichgewicht zwischen Substrat und Spaltungsprodukten führen, und stellt die gemessene Reaktionsgeschwindigkeit als Differenz beider entgegengerichteter Umsätze dar. Das führt bei der Annahme, daß die Konzentrationsänderung mit der Zeit fortwährend der Enzymintensität proportional ist, zur Gleichung

1) Das., S. 32.

2) Zeitschrift f. Elektrochemie, Bd. XI, S. 790 (1905).

3) Das., S. 793.

4) Zeitschrift f. physik. Chemie, Bd. XLIX, S. 456 (1904).

5) Zeitschrift f. physik. Chemie, Bd. LII, S. 257 (1905).

6) Das., S. 286.

7) Henri, Lois générales.

8) Das. 


$$
-\frac{d \mathrm{C}}{\mathrm{dt}}=\mathrm{I}\left[\mathrm{k}_{1} \mathrm{C}-\mathrm{k}_{2}\left(\mathrm{C}_{0}-\mathrm{C}\right)^{2}\right]
$$

$\left(\mathrm{C}_{0}\right.$ die Anfangskonzentration, $\mathrm{G}$ die Konzentration zur Zeit $\mathrm{t}$, I Intensität des Enzyms, $\mathrm{k}_{1} \mathrm{k}_{2}$ Konstante.)

Die Intensität wird dann durch empirische Formeln als Funktion von $\mathrm{C}$ und $\mathrm{C}_{0}$ ausgedrückt; ferner wird die Formel für das chemische Gleichgewicht gegeben.

Gegen diese Ausführungen kann aber einiges eingewendet werden. Einmal liegen die Gleichgewichtsbedingungen bei den Fermentreaktionen recht kompliziert; bei der Reversion scheinen sich leicht Isomere des Stoffes zu bilden, der ursprünglich gespalten wurde; ${ }^{1}$ ) außerdem treten erfahrungsmäßig die Erscheinungen des sogenannten "falschen Gleichgewichts» ein, das von der Fermentkonzentration abhängig zu sein scheint. ${ }^{2}$ ) Gerade für die Inversion des Rohrzuckers, die Hr. Visser behandelt hat, fehlt der sichere experimentelle Beweis für die Synthese bisher. Endlich führt das Formelmaterial aber gar nicht dahin, daß die endgültig erhaltene Geschwindigkeitskonstante von der Substratkonzentration unabhängig wäre (Henri). ${ }^{3}$ )

Auch auf die Studien Armstrongs $\left.{ }^{4}\right)$ möge noch hingewiesen sein.

2. Vor Vissers, Barendrechts und Henris letzter Formulierung habe ich in dieser Zeitschrift ${ }^{5}$ ) einen Versuch

2) Vgl. Zeitschrift f. allgem. Physiologie, Bd. IV, S. 193 (1904).

₹) Vgl. Tammann, 1. c.; ferner Bodenstein, Internat. Kongr. f. Chem. Rom 1906.

2) Zeitschrift f. physik. Chemie, Bd. LI, S. 26 (1905).

4) Proc. Roy. Soc., Bd. LXXIII, S. 500, 516, 526; Bd. LXXIV, S. $184,188,195$ (1904).

5) Diese Zeitschrift, Bd. XLI, S. 416 (1904); das., Bd. XLIII, S. 222 (1904). - In meiner etwas später verfaßsten Habilitationsschrift (Heidelberg, März 1905) habe ich wohl richtiger als in der Publikation statt der Viskosität $\eta$ die Durchflußzeit $\delta$ durch die Kapillaren geschrieben. Es hieß demnach: «Ist $\delta$ die Durchflußzeit einer Lösung von a durch eine Kapillare, durch welche dasselbe Volumen Wasser die Durchflußzeit 1 gebraucht, und $\mathrm{m}$ eine Konstante, so setzen wir $\mathrm{k}=\left(\frac{1}{\delta-1}\right)^{\mathrm{m}}$. Es bleibt noch die Aufgabe, $\delta$ als Funktion von a anzugeben, dazu kann die bekannte Formel $\mathrm{R}+\mathrm{Aa}+\mathrm{Ba}^{2}+\mathrm{Ca}^{3}$ dienen, wo $\mathrm{R}=1$ gesetzt ist.» 
etwas anderer Art gemacht, für die Funktion F (k, a) einen Ausdruck zu finden und auf den Anschauungen Bredigs und Nernsts fußend den ersten Weg Henris fortzusetzen. Handelt es sich bei den Fermentreaktionen wirklich um ein heterogenes Medium, dann durfte es nicht wundernehmen, wenn die einfache Form des Massenwirkungsgesetzes für homogene Lösungen nicht anwendbar erscheint!

Der Kolloidcharakter der Fermente ist bisher wohl kaum strittig, da sie die den kolloidalen Lösungen eigentümlichen irreversibeln Zustandsänderungen und anderen Eigenschaften zeigen. ${ }^{1}$ ) Ganz allgemein stellt man sich heute wohl vor, daß der Übergang von krystalloiden über kolloide Lösungen zu Suspensionen stetig sei, abhängig von der Größe der Teilchen. Für die Typen solcher Systeme sind gewisse Eigenschaften genügend charakteristisch, dagegen ist die Abgrenzung der Übergangsformen oft recht schwierig; ${ }^{2}$ ) so braucht zwischen den Eigenschaften der "Lösungen " hochmolekularer Stoffe und ultramikroskopischer Suspensionen kein Unterschied $\mathrm{zu}$ sein. Meistens nimmt man wohl an, daß die Fermentlösungen ihren Eigenschaften nach diesen Übergangsgliedern sehr nahe stehen; dann darf man aber die Eigenschaften heterogener Systeme erwarten.

Nun ist die Frage, inwieweit die Nernstsche Formel in unseren Fällen prinzipiell angewendet werden darf.

In der *Thermodynamik technischer Gasreaktionen * entwickelt F. Haber sehr klar: ${ }^{3}$ ) «Die Nernstsche Theorie der Reaktionsgeschwindigkeiten an heterogenen Flächen, die Brunner

1) Vgl. dazu Bredig, Anorganische Fermente, und Zsigmondy, Zur Kenntnis der Kolloide (1905).

2) Das., S. 10 u. ff. - Herr Euler (Diese Zeitschrift, Bd. XLV, S. 443 [1905]) hält freilich die Katalaselösungen für ein homogenes System, obschon gerade hier die Analogie mit Bredigs kolloidalem Platin in jeder Weise hervortritt. Für Bredigs Sol läßt sich die Heterogenität bekanntlich mit dem Ultramikroskop einwandsfrei beweisen, für die Fermentlösungen ist das freilich ausgeschlossen, da - solange reine Lösungen fehlen - der Einwurf berechtigt erscheint, daß Beimengungen das Sichtbare seien. - Übrigens dürfte die Anschauung Eulers heute ziemlich vereinzelt dastehen. Vgl. dazu auch Senter, Diese Zeitschrift, Bd. XLVII, S. 127 (1906).

3) S. 237. 
bestätigt hat, gilt für den Grenzfall; daß die spezifisch-chemischen Einflüsse, welche den Vorgang zu einem langsamen machen, entfallen. Sie lehrt uns, wie die Diffusionsgeschwindigkeit quantitativ die Umsatzgeschwindigkeit bestimmt, sofern die Diffusionsgeschwindigkeit die maßgebliche ist. Dies trifft immer zu, wenn die chemische Geschwindigkeit sehr groß gegen die Diffusionsgeschwindigkeit ist.» ${ }^{1}$ ) Für unsere Fälle ist es nun sehr schwierig, wenn nicht unmöglich, sich über diese Verhältnisse ein sicheres Urteil zu bilden.

Sand $^{2}$ ) und G. Senter ${ }^{3}$ ) haben sich mit der Frage in dem von Bredig untersuchten Fall beschäftigt; der wahrscheinlichste Schluß ist, daß Konvektionsströme eine wesentliche Rolle spielen und die Diffusionsgeschwindigkeit im Verhältnis zur Reaktionsgeschwindigkeit klein ist. Eine Ansicht, wie die Verhältnisse bei den Fermentreaktionen liegen, ist vorläufig schwerlich sicher zu begründen. Die Temperaturkoeffizienten der Fermentreaktionen lassen sich zur Entscheidung kaum herbeiziehen, da ja in keiner Weise vorhergesagt werden kann, wie die Bewegung (das «Rühren») der Kolloidteilchen durch die Temperatur beeinflußt werden mag. Aus ultramikroskopischen Beobachtungen, wie sie $\mathrm{Bredig}^{4}$ ) soeben mitgeteilt hat, folgt ein ganz bedeutender Einfluß. Ferner sind z. B. die Temperaturkoeffizienten der Fermentreaktionen ganz allgemein für tiefere Temperaturen bedeutend größer als für höhere. Bei mittleren Temperaturen sind gerade die Temperaturkoeffizienten der Reaktionen in Lösung ziemlich klein (was eben als Grund für Diffusionsvorgänge

1) Herr Euler (1. c., S. 442) schließt aus der Kleinheit der gemessenen Geschwindigkeitskonstanten, daß dies für die Fermentreaktionen nicht gelten könne, ein Gedankengang, den Herr Senter bereits zurückgewiesen hat. 1. c.

Auch Eulers Schluß von den Versuchen Sjöqvists auf den Verlauf in ahomogenen Systemen ist unstatthaft nach den Resultaten Dauwes (Hofmeisters Beitr., Bd. VI, S. 456 [1905]), welche über die Komplikation der hier herrschenden Verhältnisse orientieren.

$\left.{ }^{2}\right)$ Zeitschrift f. physik. Chemie, Bd. LI, S. 641 (1905).

s) Das., Bd. LII, S. 737 (1905) u. Journ. of Physic. Chem., Vol. IX, S. 311 (1905).

4) Zeitschrift für Elektrochemie, Bd. XII (Hauptvers. d. D. Bunsenges. zu Dresden 1906). 
gelten könnte), groß sind nur die Koeffizienten der Reaktionen, in denen es sich um Auflösung oder Fällung von Kolloiden handelt, sowie die der Zymasewirkung, bei der es sich aber um eine Folgereaktion handeln soll. ${ }^{1}$ )

Herr Henri ${ }^{2}$ ) hat die Anwendbarkeit der Nernstschen Formel auf Grund einer Berechnung geleugnet; durch Einsetzung der gefundenen Reaktionsgeschwindigkeiten und abgeschätzter Werte für die Oberfläche des Kolloids sowie der Diffusionskonstanten des Substrates wurden nämlich unmögliche Werte

1) Buchner und Meisenheimer, Ber. d. Deutsch. chem. Ges., Bd. XXXVII, S. 417 (1901). Daten für die Temperaturkoeff., s. Zeitschrift f. allg. Physiol., Bd. IV. l. c., auch Senter, Journ. of Physic. Chem., Bd. IX, S. 317 (1905). - Genaueres und eingehenderes experimentelles Material denke ich nächstens mitzuteilen.

2) Zeitschrift f. Elektrochem., Bd. XI, S. 790 (1905). Herr Henri erhebt (Zeitschrift f. physik. Chemie, Bd. LI, S. 27 [1905]) noch andere Einwürfe, die hier beantwortet sein mögen:

Ad 1. Statt in der Formel $\mathrm{R}+\mathrm{Aa}+\mathrm{Ba}^{2}+\mathrm{Ca}^{3} \cdot \mathrm{R}=0, \mathrm{zu}$ setzen, ist vielleicht in der Tat besser zu schreiben $\delta-1$, wie nun S. 368 Anm. 5 geschehen ist.

Ad 2. Aus den Werten A, B, C kann man, da ja die Aktivität des Enzyms variabel ist, nicht direkt die Viskositäten der entsprechenden Zuckerlösungen berechnen, wohl aber ist das Verhältnis (zur Durchflußzeit) bei verschiedenen Konzentrationen der Formel nach proportional, was aus der Tabelle S. 372 hervorgeht.

Ad 3. Nach der Annahme wird wohl der Umsatz durch Diffusion vermittelt, die Abhängigkeit der Geschwindigkeit von der Konzentration aber sollte in erster Linie von der Durchflußgeschwindigkeit der Substratlösung abhängen; d. h. also, in dem Versuch ist zwar $k$ eine Funktion der Diffusionskonstanten, der Unterschied der $\mathrm{k}$ aber weiterhin Funktion von a. Ein Widerspruch zum Fickschen Diffusionsgesetz liegt also keineswegs vor.

Ad 4. Inaktive Stoffe (Glycerin, Harnstoff, auch Zucker), welche die Viskosität erhöhen, nehmen hemmenden Einfluß auf die Reaktionsgeschwindigkeit der Fermentreaktionen (vergl. S. 372); also irrt Herr Henri, wenn er sagt, , dies ist in der Tat gar nicht der Falls.

Ad 5. Daß bei vergleichbaren heterogenen Reaktionen der Fall ebenso liegt, wie bei Fermentreaktionen (wie ich voraussagte, Habilitationsschrift), daß also die Erhöhung der Viskosität eine Hemmung hervorruft, hat Bredig soeben gezeigt. Bei $\mathrm{H}_{2} \mathrm{O}_{2}$ wurde in $\mathrm{zu}$ verdünnten Lösungen gearbeitet, als daß die Viskosität hätte eine Rolle spielen können. 
für die Dicke der Adhäsionsschicht erhalten. Dazu hat Bredig ${ }^{1}$ ) bemerkt, daß keineswegs die ganze Oberfläche des kolloidalen Platins wirksam sein muß (noch viel unwahrscheinlicher ist das für Enzyme, wo eine «Seitenkette» an der Reaktion beteiligt sein soll), außerdem ist die Rechnung nach Bredig-Teletow aber auch falsch, da Henri vergessen hat, durch das Volumen zu dividieren; geschieht das, dann entsprechen die Werte für die Schichtdicke recht gut mit den von Brunner gefundenen.

Man sieht, daß zunächst keinerlei zwingende theoretische Gründe vorliegen, von der direkten Anwendung der Nernstschen Formel abzugehen.

Für meine Annahme, daß weiterhin die Viskosität des Mediums eine wichtige Rolle spielt, sprechen eine Reihe von Beobachtungen. Die Versuche von Braeuning ${ }^{2}$ ) zeigten ganz allgemein die Hemmung der Fermentreaktionen nach Zusatz irgendwelcher indifferenter Stoffe. Einige Versuche, die ich mit (Rohrzucker - Invertin) und Glycerin angestellt hatte, ${ }^{3}$ ) ergaben in äquiviskosen Lösungen etwa gleich großen Umsatz. Ein weiterer sehr augenfälliger Beleg für meine Anschauung ergab sich ferner aus folgendem. ${ }^{4}$ ) Vergleicht man das Verhältnis $\sqrt{\frac{1}{\delta-1}}$ (worin $\delta$ die Transpirations- oder Durchflußzeit von Rohrzucker durch Kapillaren ist) und $\mathrm{k}$ die Reaktionsgeschwindigkeit für [Rohrzucker - Invertin], so ergibt sich: ${ }^{5}$ )

Für Prozent Rohrzucker

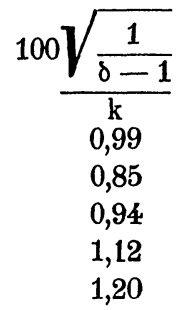

1) Zeitschrift f. Elektrochem., Bd. XI, S. 794 (1905).

2) Diese Zeitschrift, Bd. XLII, S. 70 (1904).

3) Diese Zeitschrift, Bd. XLIII, S. 222 (1904).

4) Vgl. Zeitschrift für Elektrochem., Bd. XI, S. 794 (1905).

5) $\delta$ nach Burckhard (Zeitschr. d. Ver. f. d. Rohrzucker-Industrie 1874), $\mathrm{k}$ aus den Versuchen von Henri (Zeitschrift f. physikal. Chemie, Bd. XXXIX, S. 215 (1902). Der \&Gang», den die zweite Kolonne der obigen Tabelle aufweist, ließe sich leicht erklären. 
Man sieht, der Wert des Verhältnisses ist ziemlich konstant.

$\mathrm{Daß}$ die Viskosität bei den heterogenen Reaktionen eine w'esentliche Rolle spielt und besonders bei mikroheterogenen, hat soeben auch Bredig ${ }^{1}$ ) gezeigt.

3. Und doch zwingen mich Versuche, mit deren Studium ich in Gemeinschaft mit den Herren G. Th. Becker und Dipl.-Ing. H. Kasarnowski seit längerer Zeit beschäftigt bin, der Formel, wie sie gegeben worden ist, die theoretische Bedeutung zu nehmen und sie als Interpolationsformel anzusprechen.

Hier mögen die Gründe dafür vorläufig nur angedeutet werden. Zunächst hat Herr Kasarnowski gezeigt, daß die Durchflußzeiten gleich starker Rohrzucker-und Maltoselösungen fast gleich sind; auch die Daten der anderen Zuckerlösungen weichen nicht zu stark ab. Zusätze verschiedener Zuckerarten zu [Rohrzucker - Invertin] und [Maltose - Hefenmaltase] zeigten nun eine Beeinflussung der Geschwindigkeit (in Übereinstimmung mit anderen Autoren), die nicht identisch ist mit der nach Glycerinzusatz erhaltenen.

Vor allem aber hat die Fortsetzung älterer Versuche ${ }^{2}$ ) über die Reaktion [Maltose - Hefenmaltase], die mit den genannten wieder aufgenommen wurden, eine bisher unbekannte Anomalie gezeigt. Entsprechend der S. 316 ausgesprochenen Regel nimmt die relative Reaktionsgeschwindigkeit mit Zunahme der Maltosekonzentration ab. Bei einem Gehalt von etwa $22 \%$ Maltose aber tritt in der ersten Zeit ein Minimum - eine Störung - ein, das sich späterhin wieder mehr im Sinne einer allmählich verlaufenden Kurve ausgleicht; das findet sich bei $0^{\circ}$ und bei $25^{\circ} \mathrm{C}$. Zeichnet man ferner $\mathrm{k}$ nach

$$
\frac{\mathrm{dx}}{\mathrm{dt}}=\mathrm{k}(\mathrm{a}-\mathrm{x})
$$

für gleiche $t$ als Funktion von a, so verläuft die Kurve weniger steil als bei [Rohrzucker - Invertin], was nach dem oben mit-

i) Zeitschrift f. Elektrochem., Bd. XII, Hauptvers. d. D. Bunsenges. zu Dresden.

2) Zeitschrift f. allg. Physiologie, 1. c.

Hoppe-Seyler's Zeitschrift f. physiol. Chemie. XLVIII. 
geteilten Resultat Kasarnowskis der Zurückführung der Geschwindigkeit auf die Durchflußzeiten widerspricht.

Die Reaktion (Maltose-Hefenmaltase) unterscheidet sich auch abgesehen vom Ausdruck für $\frac{\mathrm{dx}}{\mathrm{dt}}$ und der Funktion $\mathrm{F}(\mathrm{K}, \mathrm{a})$ sehr wesentlich von der Reaktion (Rohrzucker-Hefeninvertin) dadurch, daß die Geschwindigkeit des Umsatzes der Quadratwurzel der Fermentmenge ${ }^{1}$ ) proportional ist.

Nebenbei möge hier noch darauf hingewiesen sein, daß die Geschwindigkeitsmessung auch zur Prüfung der Identität quantitativ gleichartig wirkender Fermente dienen kann; die von uns untersuchte Maltase muß von der durch Henri und Philoche ${ }^{2}$ ) studierten verschieden sein.

4. Man sieht, daß trotz vielfacher Versuche eine vollständige Theorie des behandelten Problems noch nicht vorliegt. Die vorliegenden Ansätze zeigen jedenfalls bereits, daß sie sich einigermaßen einfach kaum wird gestalten lassen. Die Beziehungen zwischen stereochemischer Konfiguration des Substrats und Katalysators, die außerordentlich spezifische Wirkungsweise, ${ }^{3}$ ) die auch die Beeinflussung durch zugesetzte Stoffe zeigt, machen intermediäre Bindungen immer wahrscheinlicher. Auch die Verteilung des Substrats zwischen Lösung und Fermentteilchen spielt wohl eine wichtige Rolle, doch ist der von Henri ${ }^{4}$ ) angenommene Satz kaum richtig, daß ein Teil immer irreversibel gebunden sein müsse. Sicherlich kommt aber auch den mechanischen Vorgängen ein großer Einfluß zu. Bredigs) hat besonders anschaulich darauf hingewiesen.

Bei der Komplikation dieser Verhältnisse genügen handliche Interpolationsformeln, wie etwa die aufgestellten, dem praktischen Bedürfnis völlig.

1) Zu der von Sawjalow (Diese Zeitschrift, Bd. XLVI, S. 324 [1905]) ausgesprochenen Hypothese zur Erklärung der Schütz-Borissowschen Regel vgl. auch Höber, Phys. Chem. d. Zelle, S. 312.

2) Arch. di Fisiologia, Bd. II (1904).

8) Vgl. Bach, Ber. der Deutschen chem. Gesellsch., Bd. XXXIX, S. 2126 (1906).

4) Zeitschrift f. physik. Chemie, Bd. LI, 1. c.

5) Vgl. Zeitschrift f. Elektrochemie, Bd. XI, S. 794 (1905). 
Jedenfalls muß man aber sagen, daß ein — sagen wir so allgemeiner Satz, wie ihn Herr Euler ${ }^{1}$ ) am Schlusse seiner Zusammenstellung ausspricht, irgend eine Förderung des Problems nicht geben kann.

1) Vgl. dazu auch R. Wegscheider, Zeitschrift f. physik. Chem., Bd. XXXIX, S. 257 (1902); Bd. XLI, S. 62 (1902); ferner Bd. LI, S. 108 (1905), und Monatshefte f. Chem., Bd. XXVI, S. 1 (1905). - In derselben Publikation macht Herr Euler auch einige Bemerkungen über meine Versuche über alkoholische Gärung, die ich nicht unbeantwortet lassen darf. - Zunächst habe ich von Anfang an selbst Einwände gegen meine Versuchsanordnung gemacht (Diese Zeitschrift, Bd. XXXVII, S. 151 [1902]), besonders aber habe ich einige Monate später (s. Diese Zeitschrift, Bd. XLI, S. 417, Anm. 3) nach dem Erscheinen der Mitteilung von F. Kaufler Zeitschrift f. physik. Gh., Bd. XLIII, S. 686, 1903) versucht, mit Hefepreßsaft zu arbeiten, freilich vor der Publikation Bu chner.u. Mits cherlich(1904) über glykogenarmen Preßsaft. Daraus folgt, daß der 2 Jahre später gebrauchte Ausdruck Eulers, ich escheine zur Auffassung gekommen zu sein», daß die Acetonhefe kein günstiges Versuchsmaterial darstelle, etwas unklar gewählt ist. - Zweitens ist der Wert 2 für $n$ als der Mittelwert in der Formel $\frac{\lg k_{1}-\operatorname{lg~} k_{2}}{\lg C_{1}-\lg C_{2}}=n$ bei mir durch einen Rechenfehler entstanden, wie ich bereits in der Zeitschrift f. allg. Phys. (1904) mitgeteilt habe; bedeutet also keine Abrundung. - Wenn drittens der Verlauf der Gärung bei Eulers Versuchen regelmäßiger erscheint als bei meinen, kann das sehr wohl damit zusammenhängen, daß sich meine über bedeutend längere Zeiten erstrecken; die Parallelversuche stimmen jedenfalls recht gut überein. Im großen und ganzen führen ja auch die Versuche, die Aberson, Euler und ich ausgeführt haben, zu übereinstimmenden Resultaten. - Die davon abweichenden Versuche Slators bedürfen wohl noch der Bestätigung. 